\title{
Therapeutic implications of DNA methylation
}

\author{
Moshe Szyf \\ Department of Pharmacology \\ and Therapeutics, \\ $M c$ Gill University, \\ 3655 Sir William Osler \\ Promenade, Montreal, PQ \\ H3G 1 Y6, Canada \\ Tel.: +15143987107 \\ Fax: +15143986690 \\ moshe.szyf@mcgill.ca
}

Cancer growth and metastasis requires reprogramming of the expression of multiple genes. The epigenome, which is comprised of chromatin and the patterns of DNA methylation, sets up and maintains gene expression programs. As expected from the broad changes in gene expression in cancer, which are characterized by both silencing and activation of multiple genes, the epigenome of cancer cells is distinguished by aberration of DNA methylation patterns, which include both hypo- and hypermethylation and aberrant regulation of DNA methylation enzymes. In contrast to genetic alterations, which are fixed and are not amenable to therapeutic intervention, pharmacological agents could alter DNA methylation patterns. This raises the prospect that DNA methylation-targeted drugs will reverse cancer growth and metastasis. One of the main challenges however, is to understand the relative role of hypo- and hypermethylation in order to achieve a balance of epigenetic therapeutic agents with positive outcome and reduced adverse effects.

The epigenome directs the genome to execute multiple gene expression programs required for normal life. This exquisite programming is disrupted in cancer by either genetic mutations or epigenetic deregulation. The epigenome, as we understand it, includes chromatin which is comprised of protein structures that package the DNA in either accessible or inaccessible modes $[1,2]$, and a pattern of chemical modifications of the DNA molecule which is part of the chemical structure of the genome, DNA methylation [3]. The building block of chromatin is the nucleosome, a structure composed of an octamer of histone proteins, $\mathrm{H} 3-\mathrm{H} 4$ tetramer flanked on either side with a H2A-H2B dimer [4]. The $\mathrm{N}$-terminal tails of these histones are extensively modified by methylation [5,6], phosphorylation and acetylation $[7,8]$; and different histone variants also play a regulatory role. These modifications regulate accessibility of genes to the transcription machinery. Histone modifications are dynamic and are catalyzed by an equilibrium of enzymes such as histone acetyltransferases (HAT) which are targeted to specific genes by site-specific trans-activators and histone deacetylases (HDACs) that are recruited by transacting repressor complexes [9]. Histone modifications can act by attracting other proteins; for example, methylation at the K9 residue of H3-histone tails attracts the heterochromatin protein (HP)-1, which binds methylated histones and precipitates an inactive chromatin structure [10]. In addition to histone modifications, the positioning of nucleosomes on regulatory regions of genes is modulated by energydependent chromatin remodeling complexes [11].
There is vast literature associating different metods of chromatin modification with critical cellular processes, which is beyond the scope of this review. Different histone modifications are proposed to create a histone code, which dynamically programs and maintains gene expression profiles and is responsive to different developmental, physiological, environmental and pathological cues [1]. Therefore, it is clear why the enzymes regulating different histone modifications such as HDACs and histone methyltransferases, which involve global alteration of gene expression, are emerging as new therapeutic targets in cancer and other diseases.

A second level of epigenomic information is the DNA methylation pattern. Cytosine residues residing in the dinucleotide CGs are modified by a methyl moiety at the fifth position on the carbon ring [12]. Since not all CGs are methylated and the methylation pattern varies from cell type to cell type in a manner which correlates with the state of gene expression, it is clear that the pattern of distribution of methyl groups in different cell types carries information that either reflects or determines the unique cell-specific properties of the genome [3]. DNA methylation is an extremely stable modification and is part of the chemical structure of the genome, in contrast to chromatin, which is composed of proteins that are associated with DNA. The state of chromatin activity and DNA methylation patterns are tightly linked. It has been known for some time that open regions of chromatin are associated with hypomethylated DNA and close inactive regions of chromatin are associated with 
hypermethylated DNA [13]. DNA methylation marks silenced genes and bears the long-term memory of epigenomic programming [14,15]. Methylation of DNA adds long-term stability to states of expression, which are first defined by changes in chromatin structure (Figure 1).

Although it is clear that DNA methylation is extremely stable, it is also potentially dynamic and plastic as will be discussed below. This tension between stability and plasticity is at the heart of the pathological changes impacting on the DNA methylation pattern during cancer progression, and on the potential therapeutic opportunities in modulation of the DNA methylation machinery.

Cancer progression requires silencing of genes, which suppress tumorigenesis and

\section{Figure 1. A scheme of epigenomic states of a gene.}

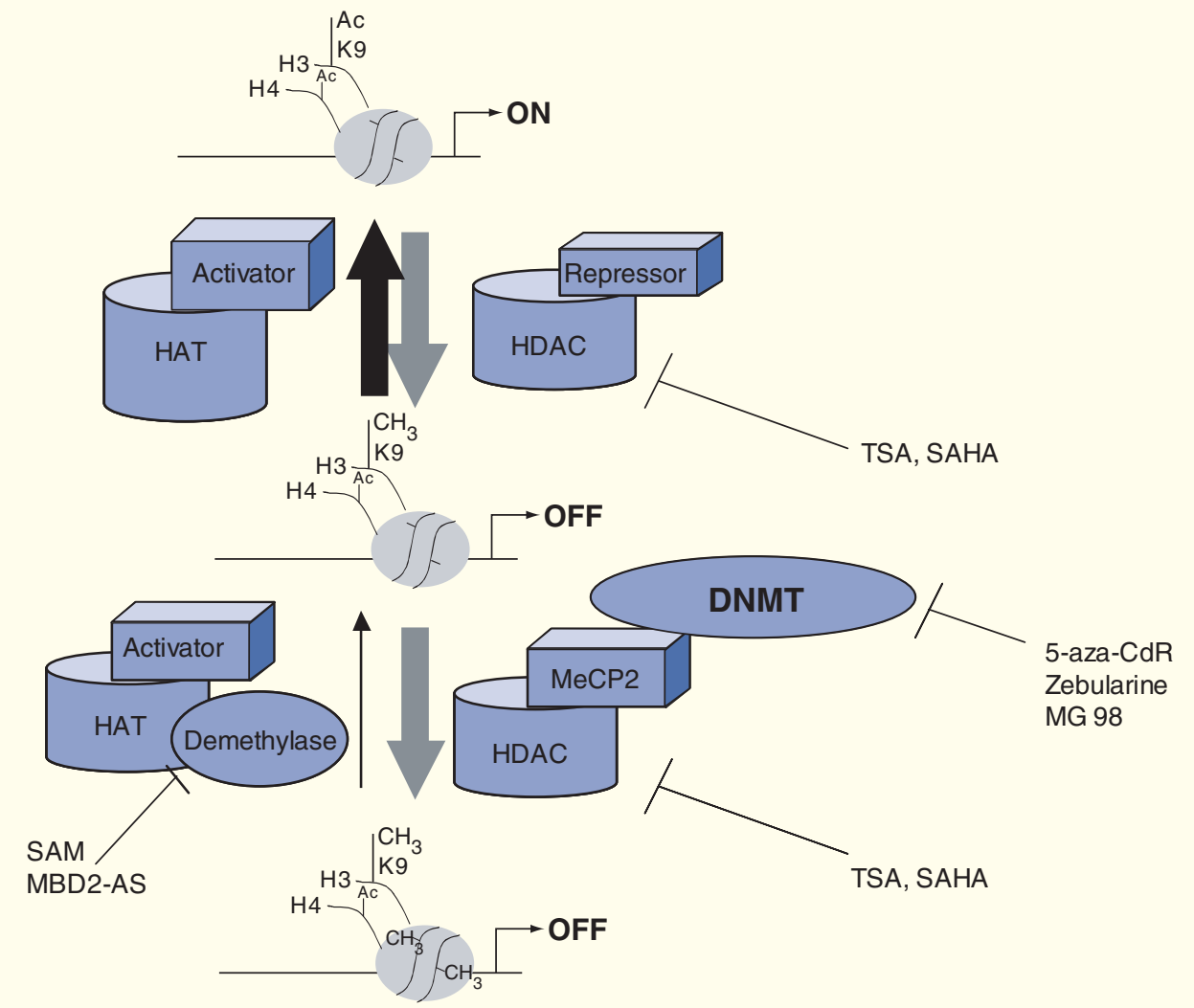

A gene can be found in different epigenomic states resulting from differences in histone modification and DNA methylation. For the sake of simplicity only histone tail lysine 9 (K9) acetylation and methylation are shown. An active gene (on) is packaged around a nucleosome with $\mathrm{K} 9$ acetylated histone tails (Ac). Histone deacetylation is catalyzed by histone deacetylases (HDACs), which are recruited by repressor complexes to specific genes. Histone acetylation is catalyzed by histone acetyl transferases (HATs), which are recruited to specific sequences by transacting activators. Binding of a repressor(s) to a gene results in loss of acetylation, increased methylation of $\mathrm{K} 9$ and silencing of gene expression (off). This is a relatively unstable silencing and is easily reversible, as indicated by the arrows. Histone deacetylases recruit DNMTs to the gene, which cause its methylation $\left(\mathrm{CH}_{3}\right)$. Methylation attracts the methyl binding protein $\mathrm{MeCP} 2$, which recruits additional corepressors and HDACs. This state of gene silencing is highly stable. Any aberrant acetylation will be countered by the HDAC recruited by MeCP2 to the methylated sequence. This state of methylation could be reversed only if acetylation is induced either pharmacologically, by TSA, or by a strong transcription factor recruiting HATs to the gene and over-riding inhibition of binding by methylation. Histone acetylation would increase the accessibility of the gene to demethylases. This event is rare but still possible. Thus, DNA methylation is a stable mark of gene silencing. The positions at which different drugs modulate the epigenome are indicated. MG 98 is a DNMT1 antisense, which is currently in clinical trials [98] and MBD2-AS is an antisense oligonucleotide targeting MBD2 [84]. DNMT: DNA methyltransferase. 
induction of genes that promote growth and metastasis. Genetic alterations such as inactivating mutations or activating mutations and rearrangements, could accomplish this switch in gene expression. However, it is also possible to alter the gene expression program using epigenomic changes. Since general enzymatic machineries control genome-wide epigenomic patterns of gene expression, a change in the DNA methylation machinery could result in variations in epigenomic programming and state of expression of numerous genes. Thus, epigenetic modulation seems to be an effective way for a cancer cell to control the state of expression of several genes concurrently. It is perhaps therefore not surprising that both hypermethylation of certain genes, which suppress tumorigenesis [16-18] and global hypomethylation [19,20], take place in cancers (Figure 2). Since the epigenome, in contrast to the genome, is reversible, it might be possible to overturn some of these epigenomic changes by targeting the DNA methylation machinery. Thus, the epigenome is a promising target for anticancer drugs. The critical question is to

Figure 2. Hypermethylation and hypomethylation of critical genes in cancer.

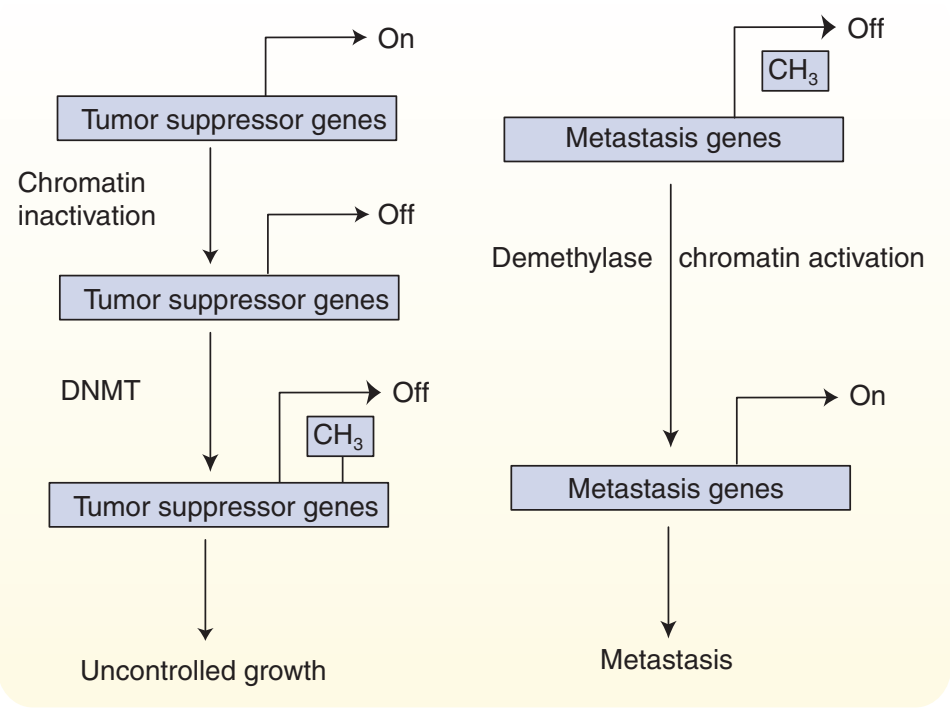

Two classes of methylation changes are encountered in cancers. Many tumor suppressor genes are hypermethylated $\left(\mathrm{CH}_{3}\right)$ and silenced. The silencing of tumor suppressors leads to uncontrolled growth. In metastatic cancer however, prometastatic genes, which are normally methylated and are methylated in nonmetastatic breast cancer, are demethylated and activated by demethylase. Inhibition of DNA methylation by DNA methylation inhibitors might lead to activation of these genes [71,80]. DNMT: DNA methyltransferase. learn how to target the required epigenetic changes to affect tumor inhibition and to steer clear of changes, which could result in promoting tumorigenesis and metastasis. In view of the fact that hypermethylation of tumor suppressor genes has been the focus recently, most attention in the field has been directed at DNA methylation inhibitors as potential activators of tumor suppressor genes. DNA methylation inhibitors are now being tested as potential anticancer agents [21,22]. However, if hypomethylation is also important for tumorigenesis, and might promote the activity of genes involved in metastasis as will be discussed below, then DNA methylation inhibition treatment might also result in the adverse effect of activation of prometastatic genes [22]. Thus, although interfering with the DNA methylation machinery is a potentially powerful approach to cancer therapy, it is imperative to gain a full understanding of the mechanisms involved in these two processes; hypermethylation and demethylation, to properly utilize agents targeting the DNA methylation machinery in anticancer therapy.

\section{DNA methylation patterns: mechanisms of formation \& maintenance DNA methyltransferases}

The transfer of the methyl group from the methyl donor $S$-adenosylmethionine (SAM) onto DNA is catalyzed by DNA methyltransferases (DNMTs) concurrently with DNA replication [23]. A number of DNMT genes and their splice variants are known in vertebrates. DNMT1 was originally proposed to be responsible for maintaining the DNA methylation pattern during cell division including cancer cells [24], while DNMT3A and DNMT3B were suggested to be responsible for de novo methylation of specific sequences during early development [25-27] as well as for maintenance of the DNA methylation of specific repetitive sequences [28,29].

\section{De novo \& maintenance DNA methylation}

It was known for some time that the DNA methylation patterns undergo dramatic changes during development and differentiation [30-32], and it was believed that the pattern of methylation is preserved through adulthood [12]. This was the common consensus, since it was thought that DNA methylation is irreversible and could only be removed by replication in the absence of DNA methyltransferase. Postmitotic nonreplicating cells would naturally preserve 
their methylation pattern. In somatic dividing cells the maintenance enzyme DNMT1 was proposed to accurately replicate the DNA methylation pattern since it could only transfer a methyl group to a hemi-methylated site, which is formed when a methylated CG sequence is replicated [33]. However, based on recent data, the idea that DNA methylation is determined exclusively by the methylation pattern of the template must be revisited. In addition, the clear differentiation between de novo and maintenance DNMT is not fully supported by recent data. Recent experiments suggests that the putative de novo enzyme DNMT3b and the proposed 'maintenance' DNMT1 interact to maintain the DNA methylation pattern [29], they are found together in multiprotein complexes [34] and that knockdown of both activities is required to cause global loss of DNMT [35-38]. Thus, the author proposes that maintenance of DNA methylation pattern is not a passive process of photocopying of a methylation pattern of a parental strand, but is rather an

\section{Figure 3. Inhibition of metastatic genes by demethylation} inhibitors.

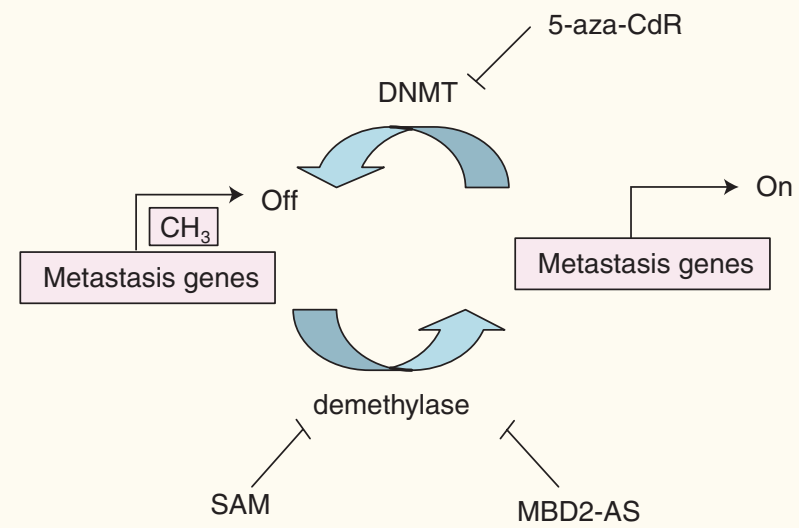

DNA demethylation agents cause activation of metastatic genes. The methylation status of metastatic genes such as UPA, is in steady state equilibrium, which is a balance of methylation catalyzed by DNMTs and demethylation catalyzed by demethylases. In metastatic cancer cells that express high demethylase activity, the balance is tilted towards demethylation. Inhibition of demethylase activity with inhibitors such as the methyl donor Sadenosylmethionine (SAM) or an antisense to MBD2 blocks demethylation and tilts the equilibrium towards the left, resulting in methylation, silencing of the genes and inhibition of metastasis [46]. In nonmetastatic cancer cells, genes required for metastasis are methylated and silenced resulting in inhibition of metastasis. Treatment of such tumors with the DNA methylation inhibitor 5aza- $C \mathrm{dR}$ results in hypomethylation, activation of metastatic genes and induction of metastasis. DNMT: DNA methyltransferase. UPA; urokinase plasminogen activator. active process of repeated dynamic decisions to methylate specific CGs. The targeting of specific DNMTs to a gene is suggested to play a critical role in determining its state of methylation. Different splice variants of all DNMTs have been identified [39,40], and some variants are more abundant in certain tumors. The repertoire of different DNMTs and their splice variants associated with a gene possibly play an important role in shaping as well as maintaining its state of methylation. DNMTs act in the context of other DNMTs and other epigenomic components. Therefore, it is proposed here that the decision to either maintain or alter a DNA methylation site is not predetermined by the original pattern of methylation. Thus, there is no inherent reason for a site to remain methylated. A methylation pattern is maintained as long as the context of DNA methylation machinery around a gene remains the same. The context of DNA methylation enzymes around a gene is defined by other transacting factors interacting with the gene and the chromatin structure. This model suggests that DNA methylation is dynamic and is not an automatic mirror image of its history. This dynamic nature of DNA methylation is consistent with the de novo methylation events observed in cancer.

\section{Demethylation \& demethylases}

If DNA methylation is dynamically maintained throughout life it should also be reversible, otherwise it will gradually drift toward increased methylation. Due to the chemical stability of DNA methylation, it has been a long-standing accepted belief that the DNA methylation pattern is a fixed characteristic of genomes in mature cells and therefore should not play a role in the dynamic changes in gene expression programming throughout adulthood. However, the author and colleagues have proposed that DNA methylation could be enzymatically reversed by demethylase activity [41] and that the DNA methylation pattern is an equilibrium of methylation and demethylation (Figure 3) [22]. Therefore, a given pattern of methylation is a steady state, which potentially could be altered by different environmental, physiological and pathological signals such as those occurring in cancer. Bhattacharya and colleagues have proposed that the methylated DNA binding protein MBD2 is a demethylase [42]. This discovery has been a matter of significant controversy since Adrian Bird's group has shown that MBD2 associates with the chromatin remodeling 
complex NurD and plays a role in the silencing of methylated genes, and they were unable to show demethylase activity for this protein [43]. Since then, evidence has been provided that MBD2 participates in active demethylation in human cells [44] by showing that antisense knockdown of this protein inhibits active demethylation [45] and results in hypermethylation of genes which are otherwise hypomethylated in cancer cells [46]. The fact that a protein, which is proposed to be a demethylase and therefore is predicted to be an activator of gene expression, also participates in gene suppression by association with a repressor complex such as NurD, seems to be paradoxical [43]. It is possible, however, to resolve this contradiction by arguing that MBD2 plays divergent roles when it is found in different promoter contexts by associating with different multiprotein machineries. In accordance with this hypothesis, Detich and colleagues showed that MBD2 demethylates in a promoter selective manner [44]. It is proposed, therefore, that MBD2 could associate with either a chromatin remodeling complex to silence genes, or serve as a demethylase associating with a different machinery.

Even if it is agreed that MBD2 bears demethylase activities, it is clear that other demethylases must exist since an $\mathrm{MBD}^{-/-}$ mouse still exhibits normal active demethylation of the paternal genome after fertilization [47].

\section{Direction of the DNA methylation equilibrium is determined by the chromatin structure of a gene}

An important question is what determines the direction of the DNA methylation equilibrium towards either methylation or demethylation? It is well established that there is a tight correlation between the state of methylation of regulatory regions of genes and their state of activity $[3,12,48]$. Methylated regulatory regions of genes are associated with inactive deacetylated chromatin while unmethylated DNA is associated with hyperacetylated open chromatin, which is transcriptionally active $[13,48]$. The relationship between DNA methylation and chromatin structure was originally believed to be unidirectional; DNA methylation determines chromatin structure. A molecular explanation for this relationship has been provided by the discovery of methylated DNA-binding proteins such as $\mathrm{MeCP} 2$ that bind methylated DNA and recruit histone deacetylases and histone methyltransferases to regulatory regions of genes, resulting in chromatin inactivation $[49,50]$. However, recent data suggests that there is a bilateral relationship between DNA methylation and chromatin structure. Inactivation of chromatin precedes and leads to DNA methylation of certain genes [51], and histone acetylation and chromatin activation bring about demethylation [52,53]. Szyf and colleagues therefore proposed that the direction of the DNA methylation equilibrium was determined by the chromatin structure of a gene. They have recently provided evidence that the DNA methylation is in a dynamic and reversible state in post mitotic tissues such as the brain long after birth, and that it could be reversed by active demethylation in response to pharmacological modulation of histone acetylation. The author and colleagues have also shown that high frequency of maternal licking and grooming could bring about demethylation of the glucocorticoid receptor in the hippocampus of the offspring early after birth at a point in time when very little DNA replication takes place. The difference in DNA methylation between high and low frequency licked offspring was maintained to adulthood. Strikingly, however, the methylation pattern could be reversed in adult rats by treatment with the histone deacetylase inhibitor trichostatin A (TSA), showing that active demethylation is induced by active chromatin in vivo [15] as it is in cell culture [52,53]. The fact that HDAC inhibitors could trigger active demethylation is of special importance in cancer therapy since HDAC inhibitors are in clinical trials as potential anticancer targets [54,55]. It is generally assumed that HDAC inhibitors only affect the state of acetylation and not DNA methylation [56]. Hypomethylation of tumor and metastasis promoting genes might be an undesirable consequence of HDAC therapy.

Although it is argued here that DNA methylation is dynamic and potentially reversible under certain conditions, it is evident that at the same time, DNA methylation is an extremely stable chemical modification of the cytosine ring, and therefore serves to stabilize silencing of genes. Therefore, it was proposed that only a persistent signal of chromatin activation would result in demethylation, whereas transient activation of chromatin would not be reflected in a change in DNA methylation [57]. Thus, DNA methylation guards the epigenome from noise but at the same time is amenable to change if the proper signals are triggered. In addition, because of the stability of the DNA methylation signal it stands to reason that it plays an important role in the stable silencing of heterochromatic regions and is recruited to these 
regions by the histone methyltransferase Suv39h and the hetereochromatin resident protein $\mathrm{HP}-1$ [58], which are highly enriched in histone $\mathrm{H} 3$ lysine 9 methylation and HP-1 proteins [5].

\section{Regional hypermethylation in cancer \& its therapeutic implications \\ Hypermethylation of tumor suppressor genes in cancer}

A large list of genes, including classical tumor suppressor genes which were defined by germline mutations as well as other genes that were shown to suppress tumor cell growth in culture, were found to be epigenetically silenced in cancer [59-61]. Most of the epigenetically silenced genes in tumors are also hypermethylated in CG sequences located in the 5' regulatory regions. The profile of methylated genes in cancers might constitute a molecular signature, which could be used to classify and stage tumors [62-64].

\section{DNA methylation \& DNMT inhibitors as anticancer agents}

Since DNA methylation can be reversed in dividing cells by inhibiting DNMTs during cell division, it was postulated that such inhibitors would cause demethylation and activation of critical tumor suppressor genes and thus block cancer cell growth. DNMTs were therefore proposed as candidate anticancer targets [21]. Nucleoside analogs of cytidine such as 5-aza-deoxy-cytidine (5-azaCdR) [65] and zebularine [66], which are incorporated into DNA, trap DNMTs during movement of the replication fork and cause passive demethylation of nascent DNA, also bring about demethylation and activation of tumor suppressor genes [67]. Since nucleoside analogs exhibit cellular toxicities, attempts are being made to identify nonnucleoside DNMT inhibitors. Nevertheless, it should be mentioned that some nucleoside analogs such as zebularine might be less toxic than others such as 5-aza-CdR [66]. There have been a number of clinical trials with either 5-aza-CdR alone or in combination with histone deacetylase inhibitors. The basic assumption of the combination therapy is that HDAC inhibitors and 5-aza$\mathrm{CdR}$ are acting independently on two different epigenetic modifications; histone acetylation and DNA methylation [56]. However, as discussed above our data suggests that the histone deacetylase inhibitor TSA could bring about demethylation of DNA as well as histone acetylation, and that these two processes are tightly related $[15,52,53]$. It is still unclear whether 5 -aza-CdR will become an effective agent in anticancer therapy.
Somewhat encouraging effects were shown in treatment of leukemias but not solid tumors. The efficacy of the combination therapy with HDAC inhibitors is not yet known [68].

To bypass the problem of nucleoside-analogassociated toxicity as well as toxicity associated with the trapping of a large complex of proteins onto DNA as is the case with 5-aza-CdR [69], Ramchandani and colleagues have developed sequence-specific DNMT1 antisense oligonucleotides [70], which were shown to inhibit tumorigenesis in murine models, and a DNMT1 antisense MG98 is now in clinical trials [68].

\section{DNA methylation inhibitors activate some prometastatic genes}

The current strategy of looking for DNMT inhibitors which cause DNA methylation inhibition focuses on the methylated tumor suppressor genes. However, one needs to consider the possibility that hypomethylation induced by either nucleoside analogs of cytidine or HDAC inhibitors will lead to activation of prometastatic genes. For example, it has been shown that 5aza-CdR, induced in a nonmetastatic human breast cancer cell line, the expression of the urokinase plasminogen activator $(u P A)$ gene encoding a protease highly implicated in breast cancer metastasis [46,71], and similarly 5-aza-CdR was shown to induce the protease heparanase implicated in metastasis [72].

\section{Methylation-independent transformation activities of DNMTI}

Data from the author's laboratory suggests that knockdown of DNMT1 by antisense inhibitors results in induction of tumor suppressors by a methylation-independent pathway [73], inhibition of DNA replication [74] and launching of an intra$S$ epigenomic damage checkpoint [75]. This treatment results in very limited DNA demethylation. The fact that antisense knock down of DNMT1 causes very limited demethylation could be explained by recent data suggesting that knock down of both DNNMT3b and DNMT1 is required to bring about significant demethylation [36], as well as by the arrest of replication by DNMT1 knock down [74] which prohibits passive demethylation. A whole-genome microarray comparison of the changes in the transcriptome caused by either inhibition of DNMT by a traditional nucleoside analog 5-aza-CdR or DNMT1 antisense, revealed that while 5-azaCdR caused activation of methylated genes such as melanoma-associated antigen gene (MAGE) 
family members, DNMT1 antisense activated predominantly ubiquitously expressed genes presumed to be unmethylated [76]. These data have two implications. First, that it is possible to bring about arrest of tumor growth by blocking the DNA methylation-independent activities of DNMT1. Second, that we need to target the DNA methylation-independent activities of DNMT1 rather than its catalytic activities. This might be a possible route for taking advantage of DNMT as an anticancer target, while avoiding the adverse effects of demethylating and activating prometastatic genes [57]. Paradoxically, we might have to look for inhibitors of DNMT1 which do not inhibit demethylation, and focus on the protein-protein interactions of DNMT1 leading to its methylation-independent effects [77].

\section{Global hypomethylation in cancer \& its therapeutic implications Causal role of hypomethylation in cancer}

An important question which has received very limited attention in recent years is whether pharmacological inhibition of the demethylation observed in cancer would be of therapeutic relevance. Global hypomethylation was implicated in chromosomal instability in cancer. This was supported by genetic evidence from hypomorphic mice with low DNMT1 expression [78], and the human ICF syndrome where a defect in DNMT3B resulted in hypomethylation and instability of the centromere-adjacent heterochromatic region (qh) of chromosomes 1, 16 and sometimes 9 [79]. Perhaps the most interesting and clinically relevant impact of hypomethylation is the well-documented regulation of prometastatic genes such as $u P A[46,71,80], s 100 A 4$ [81,82] and heparanase [72] by methylation, and their activation by hypomethylation .

Based on this data, the author and colleagues suggested that inhibition of hypomethylation should be an attractive therapeutic approach for inhibiting metastasis [83]. If demethylation plays a role in regulating multiple metastatic genes, then inhibition of demethylation should lead to concurrent suppression of metastatic genes. Although there is much mystery surrounding the enzymatic machinery involved in DNA demethylation, the author and colleagues have recently illustrated that inhibition of either MBD2, the candidate demethylase with sequence specific antisense oligonucleotides [84], or treatment with SAM, the methyl donor of the DNA methylation reaction, could lead to hypermethylation, inhibition of metastatic protein protease and inhibition of cell invasion in vitro and in vivo [46]. The author and colleagues have previously shown that SAM inhibits demethylase activity in HEK 293 cells and MBD2 demethylase activity in vitro [85]. SAM is especially interesting since its levels could be modulated by the methyl-enriching components of diets. There are animal [86-88] and some epidemiological studies correlating methyl-poor diets [89-93], and polymorphisms in methionine-synthesizing enzyme methylene tetrahydrofolate reductase (MTHFR) with some cancers [94,95]. In addition, SAM was shown to be chemoprotective against rat hepatocarcinogenesis [96] and this protection was demonstrated to involve DNA methylation since it was completely abolished by coadministration of 5-aza-CdR [97]. SAM is extremely unstable however, and can not be use clinically without modifications. However, stable analogs are available, and the auhtor's data suggests that stable analogs of SAM could be tested as antimetastatic agents. In addition, if increased SAM levels can prevent metastasis, then there might some therapeutic and prophylactic potential in methyl-enriching diets.

Since it is proposed here that DNMT1 and hypomethylation play distinct roles in tumor progression, whereas DNMT1 deregulated expression is critical for uncontrolled growth, and hypomethylation is required for invasion and metastasis, DNMT1 and DNA hypomethylation inhibitors might be used at different stages of the disease. DNMT1 inhibitors might be used to block tumor growth early in therapy and then replaced with DNA hypomethylation inhibitors in later stages when metastasis is a serious concern.

\section{Conclusion}

In summary, both global hypomethylation and regional hypermethylation confer a selective advantage upon cancer cells by targeting different sets of genes with opposing roles in cellular transformation. Regional hypermethylation targets the silencing of genes that suppress tumorigenesis, while global hypomethylation probably targets activation of genes, which are required for different stages of the transformation process. Our challenge is to learn how to selectively target these two opposite roles of DNA methylation in tumorigenesis. Although hypermethylation has been the focus of recent attention, inhibition of hypomethylation and its potential in inhibition of metastasis should be considered. 


\section{Executive summary}

The epigenome

- The epigenome directs the genome to execute gene expression programs.

- The epigenome includes chromatin and DNA methylation.

- Cytosines residing in the dinucleotide CGs are modified by a methyl moiety at the fifth position on the carbon ring.

- The pattern of DNA methylation in different cell types reflects their unique cell specific properties.

- DNA methylation is an extremely stable modification and is part of the chemical structure of the genome, in contrast to chromatin, which is composed of proteins, which are associated with DNA.

- DNA methylation patterns and chromatin structure are tightly linked.

- DNA methylation marks silenced genes and bears the long-term memory of epigenomic programming.

DNA methylation patterns: mechanisms of formation \& maintenance

- Transfer of the methyl group from methyl donor S-adenosylmethionine (SAM) onto DNA is catalyzed by DNA methyltransferases (DNMTs).

- A combination of DNMTs are involved in maintenance and generation of DNA methylation patterns.

- The DNA methylation pattern is a steady state balance of DNA methylation and demethylation reactions.

- There is a bilateral relationship between DNA methylation and chromatin structure.

- Methylated DNA binding proteins (MBD) bind methylated DNA and recruit histone deacetylases to methylated genes precipitating an inactive chromatin configuration.

- Inactive chromatin structure also leads to DNA methylation; histone deacetylases and methyl transferases recruit DNMTs to silenced chromatin resulting in DNA methylation.

- Active chromatin attracts demethylases leading to demethylation.

- MBD2 was proposed to be a DNA demethylase but the demethylase activity of MBD2 is still controversial.

- HDAC inhibitors can cause demethylation indirectly by activating chromatin and increasing accessibility to demethylases.

Regional hypermethylation in cancer \& its therapeutic implications

- A number of tumor suppressor genes are silenced by DNA methylation in cancer.

- Inhibitors of DNMT bring about passive demethylation and activation of tumor suppressor genes and possibly inhibition of tumorigenesis

- Nucleoside analog inhibitors of DNMT such as 5-aza-CdR and zebularine and DNMT antisense oligonucleotides are currently being tested in clinical trials as anticancer agents.

- Inhibition of DNA methylation can bring about the adverse effect of activation of prometastatic genes.

- DNMTs play a role in tumorigenesis, which is DNA methylation independent.

- Targeting the DNA methylation-independent functions of DNMT might be an alternative approach to DNA methylation therapy.

Global hypomethylation in cancer \& its therapeutic implications

- Hypomethylation might also play a causal role in tumorigenesis.

- Demethylase inhibitors are proposed as candidate anti-metastatic agents.

- The methyl donor SAM inhibits demethylation of the pro-metastatic gene UPA and blocks metastasis.

- SAM levels might be modulated by diet.

- MBD2 knockdown causes hypermethylation of UPA and inhibition of metastasis.

- DNA methylation therapy might involve agents which block methylation-independent functions of DNMT1 to block cell growth as well as inhibitors of demethylation to block metastasis.

\section{Future perspective}

Although there is no question that it is possible to modulate tumorigenesis and metastasis by targeting different proteins of the DNA methylation machinery, it is also clear that the involvement of DNA methylation in tumorigenesis is complex. We need to learn more about the following unresolved issues. First, we need to understand how DNMTs are targeted to specific CG islands and identify which DNMT isoforms play a role in silencing tumor suppressor genes. Second, we have to identify the transacting factors that precipitate the inactive state of chromatin associated with tumor suppressor genes which precedes hypermethylation. We then need to understand how inactive chromatin attracts DNMTs. Third, we need to increase our understanding of the methylation independent functions of DNMT.
By targeting drugs to these functions, we might be able to avoid the adverse effects associated with DNA methylation inhibitors such as activation of prometastatic genes by demethylation. Fourth, we need to define the demethylation machinery in order to identify the specific machinery responsible for hypomethylation in cancer cells. One promising target, $\mathrm{MBD} 2$, was identified, and its potential in inhibiting metastasis needs to be pursued. It is also imperative to study the potential of methyl-enriching diets and SAM analogs in inhibiting and protecting from metastasis.

\section{Acknowledgements}

The work from the author's laboratory discussed in this paper was supported by the National Cancer Institute of Canada (NCIC) and the Canadian Institute of Health Research (CIHR) 


\section{Bibliography}

Papers of special note have been highlighted as either of interest $(\bullet)$ or of considerable interest $(\bullet \bullet)$ to readers.

1. Jenuwein T, Allis CD: Translating the histone code. Science 293(5532), 10741180 (2001).

2. Strahl BD, Allis CD: The language of covalent histone modifications. Nature 403(6765), 41-45 (2000).

3. Razin A, Szyf M: DNA methylation patterns. Formation and function. Biochim. Biophys. Acta 782(4), 331-342 (1984).

4. Finch JT, Lutter LC, Rhodes D et al:: Structure of nucleosome core particles of chromatin. Nature 269(5623), 29-36 (1977).

5. Jenuwein T: Re-SET-ting heterochromatin by histone methyltransferases. Trends Cell Biol. 11(6), 266-273 (2001).

6. Rea S, Eisenhaber F, O'Carroll D et al: Regulation of chromatin structure by sitespecific histone $\mathrm{H} 3$ methyltransferases. Nature 406(6796), 593-559 (2000).

7. Wade PA, Pruss D, Wolffe AP: Histone acetylation. chromatin in action. Trends Biochem. Sci. 22(4), 128-132 (1997).

8. Wade PA, Wolffe AP: Histone acetyltransferases in control. Curr. Biol. 7(2), R82-R84 (1997).

9. Kuo MH, Allis CD: Roles of histone acetyltransferases and deacetylases in gene regulation. BioEssays 20(8), 615-626 (1998).

10. Lachner M, O'Carroll D, Rea S, Mechtler K, Jenuwein T: Methylation of histone $\mathrm{H} 3$ lysine 9 creates a binding site for HP1 proteins. Nature 410(6824), 116-120 (2001).

11. Tsukiyama T, Wu C: Chromatin remodeling and transcription. Curr. Opin. Genet. Dev. 7(2), 182-191 (1997).

12. Razin A, Riggs AD: DNA methylation and gene function. Science 210(4470), 604-610 (1980).

13. Razin A, Cedar H: Distribution of 5methylcytosine in chromatin. Proc. Natl Acad. Sci. USA 74(7), 2725-2728 (1977).

14. Novik Kl, Nimmrich I, Genc B, Maier S, Piepenbrock C, Olek A, Beck S: Epigenomics: genome-wide study of methylation phenomena. Curr. Issues Mol. Biol. 4(4), 111-128 (2002).

15. Weaver IC, Cervoni N, Champagne FA et al:: Epigenetic programming by maternal behavior. Nature Neurosci. 7(8), 847-854 (2004).

16. Baylin SB: Abnormal regional hypermethylation in cancer cells. AIDS Res. Hum. Retroviruses 8(5), 811-820 (1992).
17. Baylin SB, Hoppener JW, de Bustros A, Steenbergh PH, Lips CJ, Nelkin BD: DNA methylation patterns of the calcitonin gene in human lung cancers and lymphomas. Cancer Res. 46(6), 2917-2922 (1986).

18. Herman JG, Baylin SB: Promoter-region hypermethylation and gene silencing in human cancer. Curr. Top. Microbiol. Immunol. 249, 35-54 (2000).

19. Ehrlich M: DNA methylation in cancer: too much, but also too little. Oncogene 21(35), 5400-5413 (2002).

20. Feinberg AP, Gehrke CW, Kuo KC, Ehrlich M: Reduced genomic 5-methylcytosine content in human colonic neoplasia. Cancer Res. 48(5), 1159-1161 (1988).

21. Szyf M: DNA methylation properties. consequences for pharmacology. Trends Pharmacol. Sci. 15(7), 233-238 (1994).

22. Szyf M: Towards a pharmacology of DNA methylation. Trends Pharmacol. Sci. 22(7), 350-334 (2001).

23. Araujo FD, Knox JD, Szyf M, Price GB, Zannis-Hadjopoulos M: Concurrent replication and methylation at mammalian origins of replication. Mol. Cell. Biol. 18(6), 3475-3482 (1998).

24. Robert MF, Morin S, Beaulieu N et al:: DNMT1 is required to maintain $\mathrm{CpG}$ methylation and aberrant gene silencing in human cancer cells. Nature Genet. 33(1), 61-65 (2003).

25. Hansen RS, Wijmenga C, Luo P et al:: The DNMT3B DNA methyltransferase gene is mutated in the ICF immunodeficiency syndrome. Proc. Natl Acad. Sci. USA 96(25), 14412-14417 (1999).

26. Okano M, Bell DW, Haber DA, Li E: DNA methyltransferases Dnmt3a and Dnmt3b are essential for de novo methylation and mammalian development. Cell 99(3), 247-257 (1999).

27. Okano M, Xie S, Li E: Cloning and characterization of a family of novel mammalian DNA (cytosine-5) methyltransferases [letter]. Nature Genet. 19(3), 219-220 (1998).

28. Chen T, Ueda Y, Dodge JE, Wang Z, Li E: Establishment and maintenance of genomic methylation patterns in mouse embryonic stem cells by $D n m t 3 a$ and Dnmt3b. Mol. Cell. Biol. 23(16), 5594-5605 (2003).

29. Liang G, Chan MF, Tomigahara Y et al.: Cooperativity between DNA methyltransferases in the maintenance methylation of repetitive elements. Mol. Cell. Biol. 22(2), 480-491 (2002).

30. Cedar H, Razin A: DNA methylation and development. Biochim. Biophys. Acta 1049(1), 1-8 (1990).
31. Kafri T, Gao X, Razin A: Mechanistic aspects of genome-wide demethylation in the preimplantation mouse embryo. Proc. Natl Acad. Sci. USA 90(22), 10558-10562 (1993).

32. Razin A, Shemer R: DNA methylation in early development. Hum. Mol. Genet. 4(Spec No), 1751-1755 (1995).

33. Gruenbaum Y, Cedar H, Razin A: Substrate and sequence specificity of a eukaryotic DNA methylase. Nature 295(5850), 620622 (1982).

34. Kim GD, Ni J, Kelesoglu N, Roberts RJ, Pradhan S: Co-operation and communication between the human maintenance and de novo DNA (cytosine-5) methyltransferases. EMBO J. 21(15), 41834195 (2002).

35. Leu YW, Rahmatpanah F, Shi $\mathrm{H}$ et al.: Double RNA interference of DNMT3b and DNMT1 enhances DNA demethylation and gene reactivation. Cancer Res. 63(19), 61106115 (2003).

36. Rhee I, Bachman KE, Park BH et al.: DNMT1 and DNMT3b cooperate to silence genes in human cancer cells. Nature 416(6880), 552-556 (2002).

37. Rhee I, Jair KW, Yen RW et al.: CpG methylation is maintained in human cancer cells lacking DNMT1. Nature 404(6781), 1003-1007 (2000).

38. Ting AH, Jair KW, Suzuki H, Yen RW, Baylin SB, Schuebel KE: CpG island hypermethylation is maintained in human colorectal cancer cells after RNAi-mediated depletion of DNMT1. Nature Genet. 36(6), 582-584 (2004).

39. Deng J, Szyf M: Multiple isoforms of DNA methyltransferase are encoded by the vertebrate cytosine DNA methyltransferase gene. J. Biol. Chem. 273(36), 22869-22872 (1998).

40. Weisenberger DJ, Velicescu M, Cheng JC et al:: Role of the DNA methyltransferase variant DNMT3b3 in DNA methylation. Mol. Cancer Res. 2(1), 62-72 (2004).

41. Ramchandani S, Bhattacharya SK, Cervoni N, Szyf M: DNA methylation is a reversible biological signal. Proc. Natl Acad. Sci. USA 96(11), 6107-6112 (1999).

42. Bhattacharya SK, Ramchandani S, Cervoni $\mathrm{N}$, Szyf M: A mammalian protein with specific demethylase activity for $\mathrm{mCpG}$ DNA. Nature 397(6720), 579-583 (1999).

43. $\mathrm{Ng} \mathrm{HH}$, Zhang Y, Hendrich B et al:: MBD2 is a transcriptional repressor belonging to the MeCP1 histone deacetylase complex. Naure Genet. 23(1), 58-61 (1999).

44. Detich N, Theberge J, Szyf M: Promoterspecific activation and demethylation by 
MBD2/demethylase. J. Biol. Chem. 277(39), 35791-35794 (2002).

45. Detich N, Bovenzi V, Szyf M: Valproate induces replication-independent active DNA demethylation. J. Biol. Chem. 278(30), 27586-27592 (2003).

46. Pakneshan P, Szyf M, Farias-Eisner R, Rabbani SA: Reversal of the hypomethylation status of urokinase (uPA) promoter blocks breast cancer growth and metastasis. J. Biol. Chem. 279(30), 3173531744 (2004).

47. Oswald J, Engemann S, Lane $\mathrm{N}$ et al.: Active demethylation of the paternal genome in the mouse zygote. Curr. Biol. 10(8), 475-448 (2000).

48. Razin A: CpG methylation, chromatin structure and gene silencing - a three-way connection. EMBO J. 17(17), 4905-4908 (1998).

49. Jones PL, Veenstra GJ, Wade PA et al:: Methylated DNA and MeCP2 recruit histone deacetylase to repress transcription. Nature Genet. 19(2), 187-191 (1998).

50. Nan X, Ng HH, Johnson CA et al:: Transcriptional repression by the methylCpG-binding protein $\mathrm{MeCP} 2$ involves a histone deacetylase complex. Nature 393(6683), 386-389 (1998).

51. Bachman KE, Park BH, Rhee I et al.: Histone modifications and silencing prior to DNA methylation of a tumor suppressor gene. Cancer Cell 3(1), 89-95 (2003).

52. Cervoni N, Detich N, Seo SB, Chakravarti D, Szyf M: The oncoprotein Set/TAF-1 $\beta$, an inhibitor of histone acetyltransferase, inhibits active demethylation of DNA, integrating DNA methylation and transcriptional silencing. J. Biol. Chem. 277(28), 25026-25031 (2002).

53. Cervoni N, Szyf M: Demethylase activity is directed by histone acetylation. J. Biol. Chem. 276(44), 40778-40877 (2001).

54. Kramer OH, Gottlicher M, Heinzel T: Histone deacetylase as a therapeutic target. Trends Endocrinol. Metabol. 12(7), 294-300 (2001).

55. Weidle UH, Grossmann A: Inhibition of histone deacetylases. a new strategy to target epigenetic modifications for anticancer treatment. Anticancer Res. 20(3A), 1471-1185 (2000).

56. Cameron EE, Bachman KE, Myohanen S, Herman JG, Baylin SB: Synergy of demethylation and histone deacetylase inhibition in the re-expression of genes silenced in cancer. Nature Genet. 21(1), 103-107 (1999).
57. Szyf M: Targeting DNA methylation in cancer. Ageing Res. Rev. 2(3), 299-328 (2003).

58. Lehnertz B, Ueda Y, Derijck AA et al.: Suv39h-mediated histone H3 lysine 9 methylation directs DNA methylation to major satellite repeats at pericentric heterochromatin. Curr. Biol. 13(14), 1192-1200 (2003).

59. Baylin SB, Esteller M, Rountree MR et al.: Aberrant patterns of DNA methylation, chromatin formation and gene expression in cancer. Hum. Mol. Genet. 10(7), 687-692 (2001).

60. Esteller M, Silva JM, Dominguez G et al.: Promoter hypermethylation and BRCA1 inactivation in sporadic breast and ovarian tumors. J. Natl Cancer Inst. 92(7), 564-569 (2000).

61. Esteller M, Sparks A, Toyota M et al:: Analysis of adenomatous polyposis coli promoter hypermethylation in human cancer [In Process Citation]. Cancer Res. 60(16), 4366-4371 (2000).

62. Paz MF, Fraga MF, Avila $S$ et al:: A systematic profile of DNA methylation in human cancer cell lines. Cancer Res. 63(5), 1114-1121 (2003).

63. Shi H, Maier S, Nimmrich I et al.: Oligonucleotide-based microarray for DNA methylation analysis. principles and applications. J. Cell. Biochem. 88(1), 138-143 (2003).

64. Yan PS, Perry MR, Laux DE, Asare AL, Caldwell CW, Huang TH: CpG island arrays. an application toward deciphering epigenetic signatures of breast cancer. Clin. Cancer Res. 6(4), 1432-1438 (2000).

65. Jones PA: Altering gene expression with 5azacytidine. Cell 40(3), 485-486 (1985).

66. Cheng JC, Matsen CB, Gonzales FA et al.: Inhibition of DNA methylation and reactivation of silenced genes by zebularine. J. Natl Cancer Inst. 95(5), 399-409 (2003).

67. Merlo A, Herman JG, Mao L et al.: 5' CpG island methylation is associated with transcriptional silencing of the tumour suppressor p16/CDKN2/MTS1 in human cancers. Nature Med. 1(7), 686-692 (1995).

68. Goffin J, Eisenhauer E: DNA methyltransferase inhibitors-state of the art. Ann. Oncol. 13(11), 1699-1716 (2002).

69. Juttermann R, Li E, Jaenisch R: Toxicity of 5-aza-2'-deoxycytidine to mammalian cells is mediated primarily by covalent trapping of DNA methyltransferase rather than DNA demethylation. Proc. Natl Acad. Sci. USA 91(25), 11797-11801 (1994).

70. Ramchandani S, MacLeod AR, Pinard M, von Hofe E, Szyf M: Inhibition of tumorigenesis by a cytosine-DNA, methyltransferase, antisense oligodeoxynucleotide. Proc. Natl Acad. Sci. USA 94(2), 684-689 (1997).

71. Guo Y, Pakneshan P, Gladu J, Slack A, Szyf M, Rabbani SA: Regulation of DNA methylation in human breast cancer. Effect on the urokinase-type plasminogen activator gene production and tumor invasion. J. Biol. Chem. 277(44), 41571-41579 (2002).

72. Shteper PJ, Zcharia E, Ashhab Y et al.: Role of promoter methylation in regulation of the mammalian heparanase gene. Oncogene 22(49), 7737-7749 (2003).

73. Milutinovic S, Knox JD, Szyf M: DNA methyltransferase inhibition induces the transcription of the tumor suppressor p21(WAF1/CIP1/sdi1). J. Biol. Chem. 275(9), 6353-6359 (2000).

74. Knox JD, Araujo FD, Bigey P et al.: Inhibition of DNA methyltransferase inhibits DNA replication. J. Biol. Chem. 275(24), 17986-17990 (2000).

75. Milutinovic S, Brown SE, Zhuang Q, Szyf M: DNA methyltransferase 1 knock down induces gene expression by a mechanism independent of DNA methylation and histone deacetylation. J. Biol. Chem. 279(27), 27915-27927 (2004).

76. Milutinovic S, Zhuang Q, Niveleau A, Szyf $\mathrm{M}$ : Epigenomic stress response. Knockdown of DNA methyltransferase 1 triggers an intra-S-phase arrest of DNA replication and induction of stress response genes. J. Biol. Chem. 278(17), 14985-14995 (2003).

77. Robertson KD, Ait-Si-Ali S, Yokochi T, Wade PA, Jones PL, Wolffe AP: DNMT1 forms a complex with Rb, E2F1 and HDAC1 and represses transcription from E2F-responsive promoters. Nature Genet. 25(3), 338-342 (2000).

78. Eden A, Gaudet F, Waghmare A, Jaenisch R: Chromosomal instability and tumors promoted by DNA hypomethylation. Science 300(5618), 455 (2003).

79. Ehrlich M: The ICF syndrome, a DNA methyltransferase 3B deficiency and immunodeficiency disease. Clin. Immunol. 109(1), 17-28 (2003).

80. Pakneshan P, Xing RH, Rabbani SA: Methylation status of uPA promoter as a molecular mechanism regulating prostate cancer invasion and growth in vitro and in vivo. FASEB J. 17(9), 1081-1088 (2003).

81. Nakamura N, Takenaga K: Hypomethylation of the metastasisassociated S100A4 gene correlates with gene activation in human colon adenocarcinoma cell lines. Clin. Exp. Metastasis 16(5), 471-479 (1998). 
82. Rosty C, Ueki T, Argani P et al:: Overexpression of S100A4 in pancreatic ductal adenocarcinomas is associated with poor differentiation and DNA hypomethylation. Am. J. Pathol. 160(1), 45-50 (2002).

83. Szyf M, Pakneshan P, Rabbani SA: DNA demethylation and cancer. therapeutic implications. Cancer Lett. 211(2), 133-143 (2004).

84. Campbell PM, Bovenzi V, Szyf M: Methylated DNA-binding protein 2 antisense inhibitors suppress tumourigenesis of human cancer cell lines in vitro and in vivo. Carcinogenesis 25(4), 499-507 (2004).

85. Detich N, Hamm S, Just G, Knox JD, Szyf M: The methyl donor Sadenosylmethionine inhibits active demethylation of DNA. A candidate novel mechanism for the pharmacological effects of $S$-adenosylmethionine. J. Biol. Chem. 278(23), 20812-20820 (2003).

86. Bhave MR, Wilson MJ, Poirier LA: $c-H$-ras and $c-K$-ras gene hypomethylation in the livers and hepatomas of rats fed methyldeficient, amino acid-defined diets. Carcinogenesis 9(3), 343-348 (1988).

87. Kanduc D, Ghoshal A, Quagliariello E, Farber E: DNA hypomethylation in ethionine-induced rat preneoplastic hepatocyte nodules. Biochem. Biophys. Res. Commun. 150(2), 739-744 (1988).
88. Wilson MJ, Shivapurkar N, Poirier LA; Hypomethylation of hepatic nuclear DNA in rats fed with a carcinogenic methyldeficient diet. Biochem. J. 218(3), 987-990 (1984).

89. Eichholzer M, Luthy J, Moser U, Fowler B: Folate and the risk of colorectal, breast and cervix cancer. the epidemiological evidence. Swiss Med. Wkly 131 (37-38), 539-549 (2001).

90. Fang JY, Xiao SD: Folic acid, polymorphism of methyl-group metabolism genes, and DNA methylation in relation to GI carcinogenesis. J. Gastroenterol. 38(9), 821-829 (2003).

91. Giovannucci E, Rimm EB, Ascherio A, Stampfer MJ, Colditz GA, Willett WC: Alcohol, low-methionine--low-folate diets, and risk of colon cancer in men. J. Natl Cancer Inst. 87(4), 265-273 (1995).

92. Giovannucci E, Stampfer MJ, Colditz GA et al:: Folate, methionine, and alcohol intake and risk of colorectal adenoma. J. Natl Cancer Inst. 85(11), 875-884 (1993).

93. Potter JD: Methyl supply, methyl metabolizing enzymes and colorectal neoplasia. J. Nutr. 132(8 Suppl.), 2410S2412S (2002).

94. Friso S, Choi SW, Girelli D et al.: A common mutation in the 5,10methylenetetrahydrofolate reductase gene affects genomic DNA methylation through an interaction with folate status. Proc. Natl Acad. Sci. USA 99(8), 5606-5611 (2002).
95. Heijmans BT, Boer JM, Suchiman HE et al:: A common variant of the methylenetetrahydrofolate reductase gene (1p36) is associated with an increased risk of cancer. Cancer Res. 63(6), 1249-1253 (2003).

96. Pascale RM, Simile MM, De Miglio MR et al:: Chemoprevention by $S$-adenosyl-Lmethionine of rat liver carcinogenesis initiated by 1,2-dimethylhydrazine and promoted by orotic acid. Carcinogenesis 16(2), 427-430 (1995).

97. Pascale R, Simile MM, Ruggiu ME et al.: Reversal by 5 -azacytidine of the $S$-adenosylL-methionine-induced inhibition of the development of putative preneoplastic foci in rat liver carcinogenesis. Cancer Lett. 56(3), 259-265 (1991).

98. Fournel M, Sapieha P, Beaulieu N, Besterman JM, MacLeod AR: Downregulation of human DNA-(cytosine-5) methyltransferase induces cell cycle regulators p16(ink4A) and p21(WAF/Cip1) by distinct mechanisms. J. Biol. Chem. 274(34), 24250-24256 (1999).

\section{Affiliation}

- Moshe Szyf

Department of Pharmacology and Therapeutics, McGill University, 3655 Sir William Osler Promenade, Montreal, PQ H3G 1YG, Canada Tel: +15143987107

Fax: +15143986690

moshe.szyf@mcgill.ca 